\title{
SIMULATION NUMÉRIQUE D'ÉCOULEMENTS DE FLUIDES VISCOÉLASTIQUES DANS DES FILIĖRES AXISYMÉTRIQUES
}

\author{
Y. BEREAUX, J.R. CLERMONT
}

\begin{abstract}
Nous nous intéressons ici à la modélisation numérique des écoulements de fluides viscoélastiques à formulation intégrale. Ces lois de comportement sont représentatives de toute une gamme de polymères à l'état fondu. La Méthode des Tubes de Courant est particulièrement bien adaptée à ce type de problème. L'hypothèse fondamentale est de poser comme inconnue l'existence d'une fonction de transformation $f$, qui fait passer du domaine physique à un domaine transformé simple où les lignes de courant sont rectilignes et parallèles. Cette propriété simplifiera considérablement les problèmes de suivi de particule, de même que la possibilité d'un calcul par sous-domaines réduira considérablement les coûts de calcul. Dans le cadre des filières axisymétriques, des calculs ont été effectués, avec une géomètrie convergente d'angle $45^{\circ}$ avec des modèles spécifiques, comportant des comparaisons avec des résultats expérimentaux. Dans tous les cas envisagés, de hauts régimes d'écoulement, critiques pour les formulations classiques, ont été atteints sans difficultés majeures. L'examen du premier tube de courant calculé montre des effets viscoélastiques caractérisés au voisinage de la paroi et de la contraction de la filière.
\end{abstract}

Mots-clés : Simulation Numérique; Viscoélasticité; Méthode des Tubes de Courant; Modèle intégral codéformationnel; Filière convergente axisymétrique.

Classification mathématique : 76M25 Others Numerical Methods, 76A10 VISCOELASTIC FLUIDS

\section{INTRODUCTION}

Ces dernières années ont vu les progrès considérables de la simulation numérique des écoulements de fluides viscoélastiques. Néanmoins, la simulation numérique s'avère plus délicate pour les fluides décrits par des modèles intégraux qui sont d'un usage croissant dans les caractérisations expérimentales des polymères fondus. En effet, pour le calcul du tenseur des contraintes, ces modèles imposent un suivi de la particule matérielle et la connaissance des déformations subies au cours de son mouvement. La méthode des tubes de courant est particulièrement adaptée à ce type de problème. Dans un premier temps, nous en rappellerons brièvement les fondements puis nous verrons comment sont formalisés les lois de comportement intégrales codéformationnelles. Ensuite, seront exposés les schémas de discrétisation appliqués aux inconnues et aux équations gouvernantes. Enfin, des résultats numériques et des comparaisons avec les mesures experimentales correspondant à des écoulements de polyéthylènes basse densité à l'état fondu dans une filière convergente axisymétriques seront présentés. 


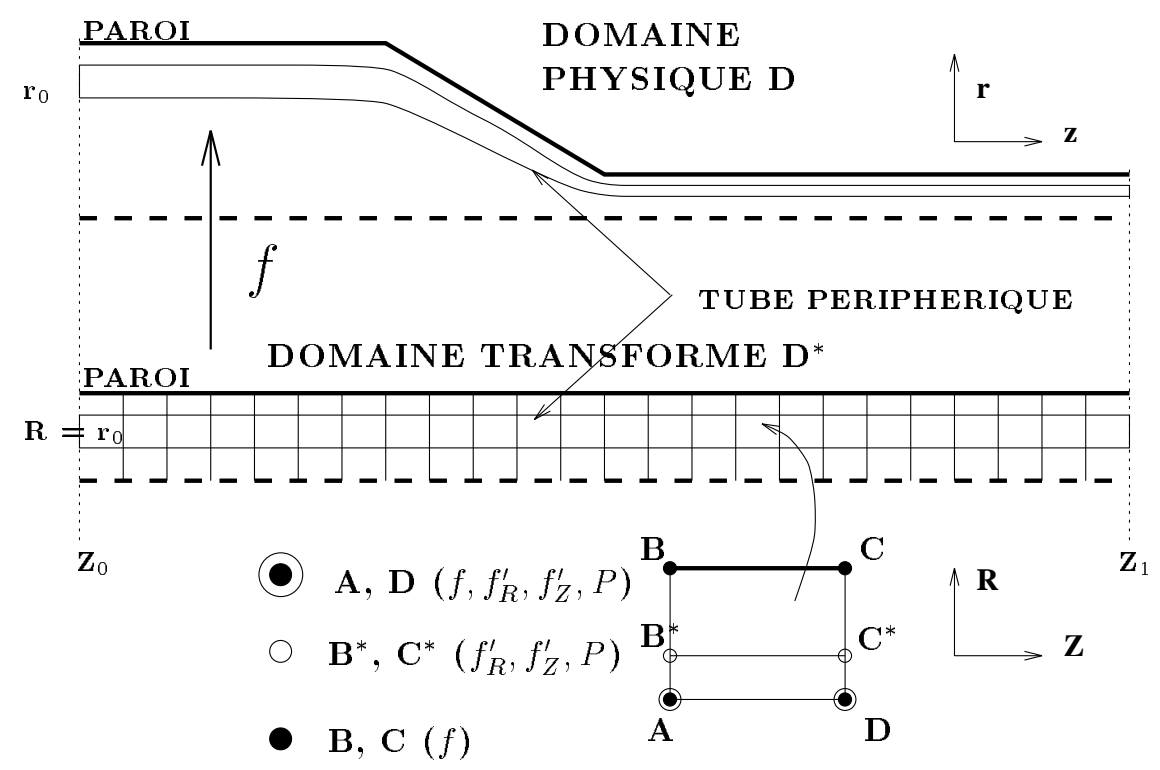

ELEMENT D'HERMITE MODIFIE

Figure 1. Transformation du domaine physique. Discrétisation du tube de courant périphérique en éléments d'Hermite modifiés.

\section{Méthode des Tubes de Courant}

\subsection{Hypothèses}

La Méthode des Tubes de Courant, initialement développée par J. R. Clermont [4], a été successivement appliquée à des écoulements de fluides très divers en relation avec des problèmes en dimension deux ou trois [2]. Dans le cas de géométries associées à des écoulements bidimensionnels, la Méthode des Tubes de Courant pose comme hypothèse fondamentale l'existence d'une fonction $f$ qui transforme le domaine physique d'écoulement $D$ en un domaine plus simple $D^{*}$ où les lignes de courant sont rectilignes et parallèles. Cette transformation est illustrée dans la Fig. 1. Cette fonction $f$ sera prise comme inconnue du problème initial à résoudre dans ce nouveau domaine, géométriquement beaucoup plus simple. Elle définit par changement de variables un nouveau repère de coordonnées curvilignes $(R, Z)$ tel que :

$$
\left\{\begin{array}{l}
r=f(R, Z) \\
z=Z
\end{array}\right.
$$

avec la condition aux limites sur une section à l'amont $z=z_{0}$, où l'écoulement est supposé connu :

$$
R=f\left(R, z_{0}\right)
$$

De plus la condition d'univocité sur la transformation impose au jacobien $J$ de celle-ci d'être non-nul en tous points du domaine, ce qui concrètement exclu de prendre explicitement en compte les zones de recirculations dans l'écoulement (3).

$$
J=\left|\frac{\partial(r, z)}{\partial(R, Z)}\right|=f_{R}^{\prime}>0
$$




\subsection{PROPRIÉTÉS}

Dans la base naturelle $\left(\vec{g}_{R}, \vec{g}_{Z}\right)$ associée à $(R, Z)$, le champ de vitesse s'exprime comme :

$$
\vec{V}=-\frac{\bar{\Psi}^{\prime}(R)}{f f_{R}^{\prime}} \vec{g}_{Z}=-\frac{f_{Z}^{\prime}}{f f_{R}^{\prime}} \bar{\Psi}^{\prime}(R) \vec{e}_{r}-\frac{1}{f f_{R}^{\prime}} \bar{\Psi}^{\prime}(R) \vec{e}_{z}
$$

oì $\bar{\Psi}^{\prime}(R)$ désigne la dérivée de la fonction de courant constante le long d'une ligne de courant et ne dépendant donc que de $R$. Cette définition de la vitesse vérifie automatiquement la condition d'incompressibilité.

\subsection{Equations gouvernantes}

Compte tenu des remarques et définitions précédentes, les équations gouvernantes qui définissent le problème sont alors :

- Les équations de la dynamique, dans le cas d'un écoulement stationnaire axisymétrique sans forces de volume ni termes d'inertie, s'écrivent suivant les variables $R, Z$ dans la base des coordonnées cylindriques $\left(\vec{e}_{r}, \vec{e}_{z}\right)$ :

$$
\begin{aligned}
& -\frac{1}{f_{R}^{\prime}} \frac{\partial P}{\partial R}+\frac{1}{f_{R}^{\prime}} \frac{\partial T^{r r}}{\partial R}-\frac{f_{Z}^{\prime}}{f_{R}^{\prime}} \frac{\partial T^{r z}}{\partial R}+\frac{\partial T^{r z}}{\partial Z}+\frac{T^{r r}-T^{\theta \theta}}{f}=0 \\
& \frac{f_{Z}^{\prime}}{f_{R}^{\prime}} \frac{\partial P}{\partial R}-\frac{\partial P}{\partial Z}+\frac{1}{f_{R}^{\prime}} \frac{\partial T^{r z}}{\partial R}-\frac{f_{Z}^{\prime}}{f_{R}^{\prime}} \frac{\partial T^{z z}}{\partial R}+\frac{\partial T^{z z}}{\partial Z}+\frac{T^{r z}}{f}=0
\end{aligned}
$$

- Une équation intégrale (6) qui traduit l'action du domaine d'écoulement complémentaire au tube de courant considéré et permet ainsi de mener la résolution par sous-domaines, en partant d'un tube de courant périphérique proche de la paroi [5].

$$
\left(\int_{S}(-P I+T) \bullet d \vec{S}\right) \bullet \vec{e}_{z}=0
$$

oì $S$ désigne la surface du tube de courant considéré.

- Des conditions aux limites sur les sections amont et aval : Ecoulement de Poiseuille

On aboutit ainsi à une formulation primaire où les seules inconnues sont $f$ et la pression $P$, dans la mesure où le tenseur des contraintes s'exprime explicitement en fonction de $f$ et de ses dérivees partielles.

\section{ModÈle intÉGRAL CODÉFORMATIONNEL}

\subsection{Modèle de Wagner}

Le modèle intégral codéformationnel utilisé est de type Wagner [9] et se présente sous la forme d'une intégrale dépendant de toute l'histoire des déformations subies et mesurées par le tenseur de Finger $\mathbf{B}(7)$. La fonction scalaire désigne le produit d'une fonction mémoire $m$ dépendant uniquement du temps et d'une fonction d'amortissemnt $h$ dépendant des premier et second invariants $I_{1}$ et $I_{2}$ du tenseur de Finger $\mathbf{B}$.

$$
\boldsymbol{T}(t)=\int_{-\infty}^{t} m\left(t-t^{\prime}\right) h\left(I_{1}, I_{2}\right) \boldsymbol{B}_{t}\left(t^{\prime}\right) d t^{\prime}
$$




\begin{tabular}{|l|l|l|}
\hline Amortissement & \multicolumn{2}{|c|}{ Mémoire } \\
\hline \multirow{4}{*}{$\mathrm{a}=0.084$} & $\lambda_{l}(\mathrm{~s})$ & $\eta_{l}=a_{l} l_{l}(\mathrm{~Pa} . \mathrm{s})$ \\
\cline { 2 - 3 } & $6.4510^{-4}$ & $9.81010^{1}$ \\
\cline { 2 - 3 } & $5.3510^{-3}$ & $2.85510^{2}$ \\
\cline { 2 - 3 } & $2.8510^{-2}$ & $7.67910^{2}$ \\
\cline { 2 - 3 } $\mathrm{b}=0.019$ & $1.5510^{-1}$ & $2.61910^{3}$ \\
\cline { 2 - 3 } $\mathrm{b}=2.06$ & $8.9110^{-1}$ & $6.46410^{3}$ \\
\cline { 2 - 3 } & $4.5810^{0}$ & $1.21910^{4}$ \\
\cline { 2 - 3 } & $2.3410^{1}$ & $1.32510^{4}$ \\
\cline { 2 - 3 } & $1.1810^{2}$ & $7.29610^{3}$ \\
\hline
\end{tabular}

TABLE 1. Paramètres matériels (PEBD $\left.160^{\circ} \mathrm{C}\right)$

\begin{tabular}{|l|l|l|}
\hline Amortissement & \multicolumn{2}{|c|}{ Mémoire } \\
\hline \multirow{4}{*}{$\mathrm{a}=0.086$} & $\lambda_{l}(\mathrm{~s})$ & $\eta_{l}=a_{l} \lambda_{l}(\mathrm{~Pa} . \mathrm{s})$ \\
\cline { 2 - 3 } & $1.2810^{-4}$ & $2.36710^{2}$ \\
\cline { 2 - 3 }$\beta=0.02$ & $6.1210^{-3}$ & $1.34610^{3}$ \\
\cline { 2 - 3 } & $4.1010^{-2}$ & $3.36310^{3}$ \\
\cline { 2 - 3 } $\mathrm{b}=2.5710^{-1}$ & $4.69110^{3}$ \\
\cline { 2 - 3 } & $2.0110^{0}$ & $3.72610^{3}$ \\
\cline { 2 - 3 } & $1.5710^{1}$ & $2.00710^{3}$ \\
\cline { 2 - 3 } & $1.3510^{2}$ & $9.56310^{2}$ \\
\hline
\end{tabular}

TABle 2. Paramètres matériels (PEBDL $160^{\circ} \mathrm{C}$ )

La fonction $m(8)$ est prise sous forme d'un spectre discret de temps et de modules de relaxation notés respectivement $\lambda_{l}$ et $a_{l}$.

$$
m\left(t-t^{\prime}\right)=\sum_{l=1}^{l=n} \frac{a_{l}}{\lambda_{l}} \exp \left(-\frac{t-t^{\prime}}{\lambda_{l}}\right)
$$

La fonction d'amortissement $h$ est prise sous une forme sigmoïdale modifiée [7], soit :

$$
h\left(I_{1}, I_{2}\right)=\frac{1}{1+a\left(\beta I_{1}+(1-\beta) I_{2}\right)^{b / 2}}
$$

Outre le spectre des temps et modules de relaxation, ce modèle fait apparaître trois paramètres matériels $a, \beta$ et $b$ qui ont été determinés expérimentalement [8] pour différents matériaux polymères, à savoir deux polyéthylènes basse densité, linéaire et branché (PEBDL, PEBD). Les valeurs des paramètres pour ces deux produits sont reportées dans les tableaux Tab. 1 et 2

\subsection{Calcul des déformations et des contraintes}

La Méthode des Tubes de Courant permet d'envisager très simplement le double problème du repérage des positions des particules sur leur trajectoire et du calcul des tenseurs des déformations entre deux positions successives d'une particule sur cette trajectoire. En effet, en se basant sur le système de coordonnées curvilignes (1) on obtient une expression analytique du tenseur gradient de déformation $\mathbf{F}$ en fonction du champ de vitesse (4) $[2,3]$. 


$$
\boldsymbol{F}^{-1}=\left(\begin{array}{ccc}
1 & 0 & 0 \\
0 & 1 & 0 \\
\frac{\partial Z}{\partial R^{\prime}} & 0 & \frac{\partial Z}{\partial Z^{\prime}}
\end{array}\right)
$$

avec :

$$
\begin{aligned}
& \frac{\partial Z}{\partial R^{\prime}}=V(R, Z) \int_{Z^{\prime}}^{Z} \frac{\partial V}{\partial R}(R, \xi) \frac{d \xi}{V^{2}(R, \xi)} \\
& \frac{\partial Z}{\partial Z^{\prime}}=\frac{V(R, Z)}{V\left(R, Z^{\prime}\right)}
\end{aligned}
$$

où $(R, Z)$ sont relatifs à la configuration actuelle de référence, $\left(R^{\prime}, Z^{\prime}\right)$ sont relatifs à la configuration déformée. Le tenseur de Finger s'écrit en notation intrinsèque comme le produit une fois contracté du tenseur gradient de déformation $\mathbf{F}^{-1}$ et de son transposé

$$
\boldsymbol{B}=\boldsymbol{F}^{-1} \bullet \boldsymbol{F}^{-1 t}
$$

Le tenseur des contraintes ( 7 ) est alors ensuite évalué au moyen d'une formule de Gauss-Laguerre à six points.

\section{SCHÉmAS DE DISCRÉTISATION}

Les schémas de discrétisation retenus concernent les fonctions $f$ et $P$ inconnues primaires du problème ainsi que les opérateurs de dérivation sur $P$ et sur le tenseur des contraintes $\mathbf{T}$ qui figurent dans les équations d'équilibre (5).

La nécessité d’interpoler précisement $f$ et ses dérivées partielles nous a conduit à définir un élément fini d'interpolation spécifique, de type Hermite faisant intervenir douze valeurs nodales $\left(f, f_{R}^{\prime}, f_{Z}^{\prime}\right)$, réparties sur six noeuds géométriques ( $\left.\mathrm{A}, \mathrm{B}, \mathrm{C}, \mathrm{D}, \mathrm{B}^{*}, \mathrm{C}^{*}\right)$ ( $\mathrm{cf}$. Fig. 1). Il s'agit donc d'un élément d'interpolation d'ordre quatre $[1,2]$.

Pour les dérivées partielles de $P$ et $\mathbf{T}$ figurant dans les équations d'équilibre nous avons adopté un schéma centré en $R$ et un schéma à 3 points en $Z$, faisant intervenir les valeurs nodales de $P$ et des calculs de $\mathbf{T}$ dans trois éléments successifs. Ces deux types d'approximation sont d'ordre deux et ont été retenus après vérification d'un critère de consistance pour un écoulement radial et d'un critère de stabilité pour un écoulement de Poiseuille perturbé [2].

La résolution du système discret non-linéaire, issu de ces procédures de discrétisation, fait alors l'objet d'un problème d'optimisation sans contraintes et est effectuée par un algorithme de région de confiance [2].

\section{RÉsultats numÉRiQues}

La simulation numérique a été menée pour des écoulements de PEBD et PEBDL (cf. Tab. 1 et 2 ) en filière convergente, avec les mêmes dimensions que celles utilisée en rhéométrie capillaire pour les mesures expérimentales de pertes de charge totale et d'entrée [8]. Les dimensions caractéristiques de la filière sont rappelés dans le tableau Tab. 3.

Les différents régimes d'écoulements étudiés peuvent être répertoriés suivant le taux de cisaillement apparent $\Gamma$, défini par :

$$
\Gamma=\frac{4 Q}{\pi R_{1}^{3}}
$$




\begin{tabular}{|l|l|}
\hline$R_{1}$ aval & $0.6250 \mathrm{~mm}$ \\
\hline$R_{2}$ amont & $4.7625 \mathrm{~mm}$ \\
\hline$L_{2} / 2 R_{1}$ amont & 9.7 \\
\hline$L_{1} / 2 R_{1}$ aval & 19.23 \\
\hline demi-angle & $45^{\circ}$ \\
\hline rapport de contraction & 7.62 \\
\hline
\end{tabular}

TABle 3. Dimensions de la filière

\begin{tabular}{|l|r|r|}
\hline$\Gamma \mathrm{s}-1$ & $S_{R}$ PEBDL & $S_{R}$ PEBD \\
\hline 3.72 & 0.432 & 1.45 \\
\hline 12.38 & 0.577 & 1.73 \\
\hline 37.16 & 0.771 & 1.97 \\
\hline 123.9 & 0.918 & 2.24 \\
\hline
\end{tabular}

TABle 4. $S_{R}$ suivant $\Gamma$ pour le PEBD et le PEBDL

où $Q$ désigne le débit, $R_{1}$ le rayon du capillaire. On peut également calculer pour chaque modèle le nombre adimensionnel $S_{R}$ qui mesure le rapport des effets élastiques aux effets visqueux à la paroi du capillaire :

$$
S_{R}=\frac{N_{1}}{2 T^{r z}}
$$

où $N_{1}$ désigne la première différence des contraintes normales, $T^{r z}$ la composante tangentielle des contraintes, calculées à la paroi du capillaire, en écoulement de Poiseuille. Les valeurs de $S_{R}$, calculés aux différents $\Gamma$ pour les deux modèles envisagés sont rassemblées dans le tableau Tab. 4. On peut noter le caractère nettement plus élastique du PEBD par rapport au PEBDL.

L'examen d'une ligne de courant $\mathrm{d} u$ tube périphérique révèle des effets viscoélastiques croissant avec les régimes d'écoulement et se traduisant par l'évolution de la position de ces lignes de courant de même origine à l'amont (cf. Fig. 2 et 3 ). L'évolution des contraintes le long d'une ligne de courant se traduit par la présence des pics de contraintes au voisinage de la section de contraction et une relaxation en aval de cette section de contraction. La contrainte de cisaillement $T^{r z}$ peut présenter un pic localisé avant la section de contraction et une relaxation de celle-ci assez rapide à l'aval (cf. Fig. 4) La différence des contraintes normales $T^{z z}-T^{r r}$ qui correspond à $N_{1}$ en écoulement de Poiseuille à l'aval (cisaillement simple), présente un pic de contrainte à la section de contraction et une relaxation à l'aval de celle-ci, beaucoup plus lente pour le PEBD que pour le PEBDL (cf. Fig. 5). Enfin, la différence des contraintes normales $T^{r r}-T^{\theta \theta}$ (qui correspond à $N_{2}$ en écoulement de Poiseuille à l'aval) est toujours négligeable devant les autres composantes du tenseur des contraintes (cf. Fig. 5)

Deux types de mesure des pertes de charge sont envisageables

- La perte de charge d'entrée $\Delta P_{e}$, qui mesure directement l'influence de la région convergente de la filière sur la pression.

- La perte de charge totale $\Delta P_{t}$ à l'extrémité de la filière, qui donne le bilan global de la chute de pression dans la filière. 
SIMULATION NUMÉRIQUE D'ÉCOULEMENTS DE FLUIDES VISCOÉLASTIQUES159
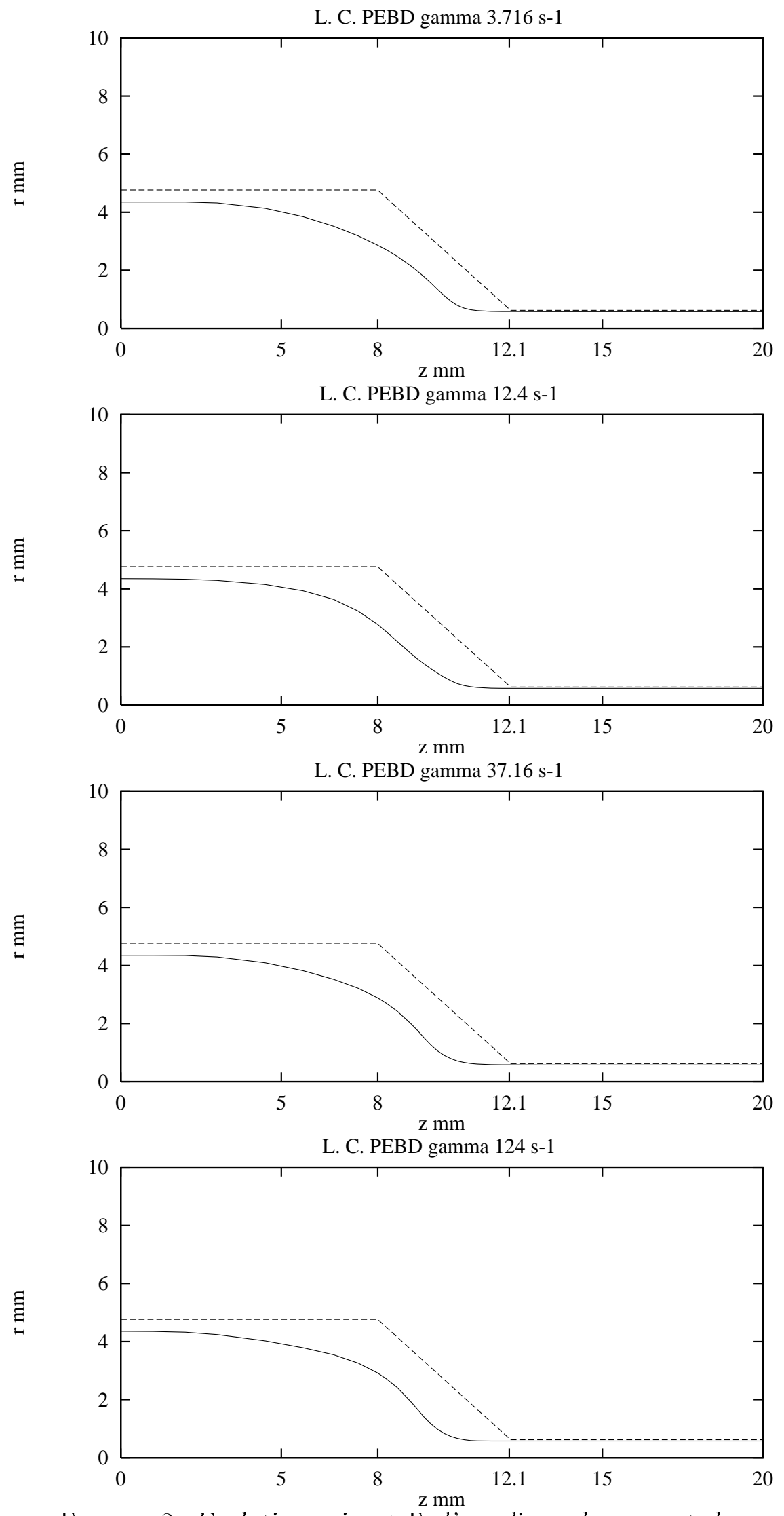

Figure 2. Evolution suivant $\Gamma_{\Gamma}^{\mathrm{z}} \mathrm{d}$ 'une ligne de courant $d u$ tube périphérique. Cas du PEBD. 

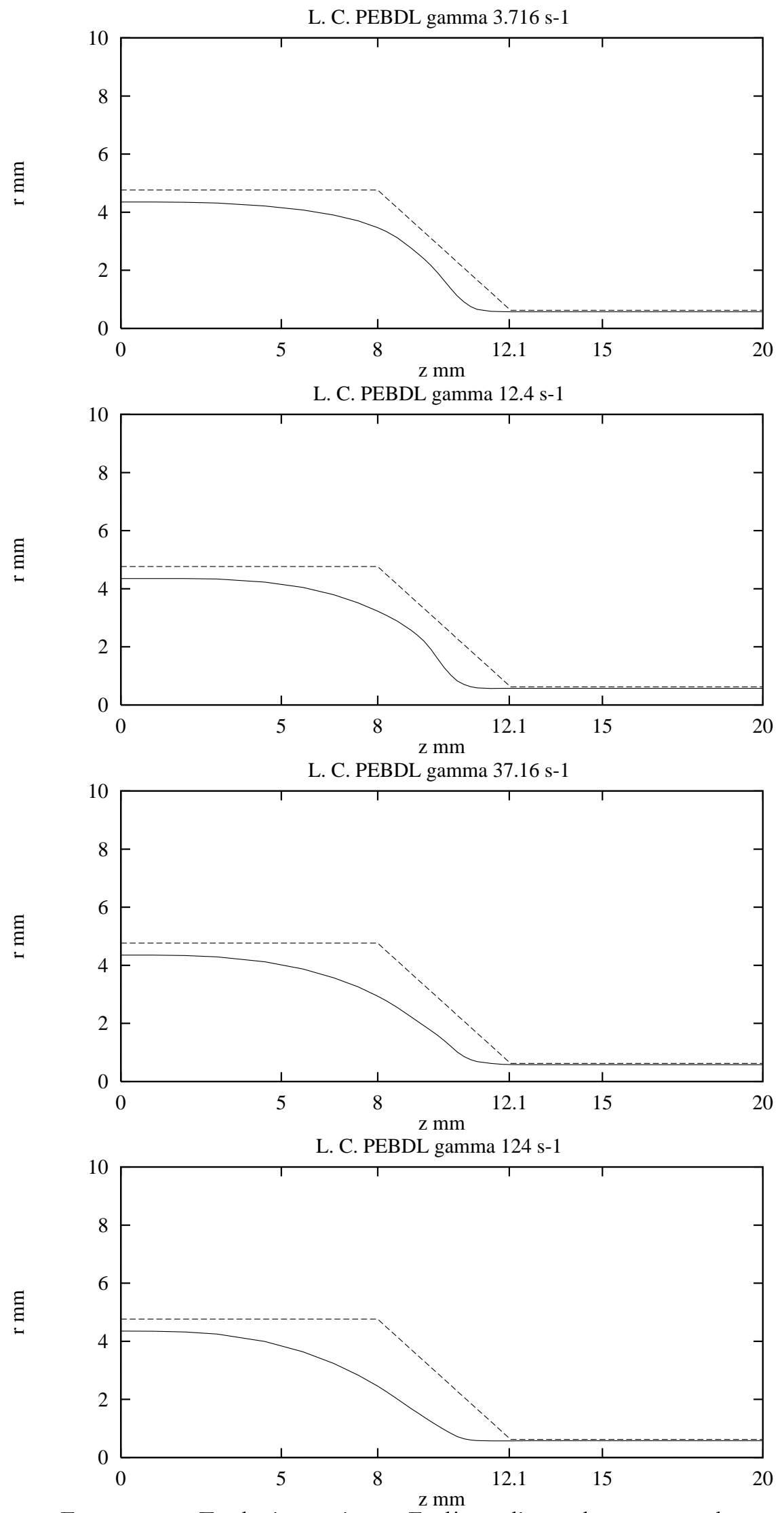

Figure 3. Evolution suivant $\Gamma$ 'une ligne de courant $d u$ tube périphérique. Cas du PEBDL. 


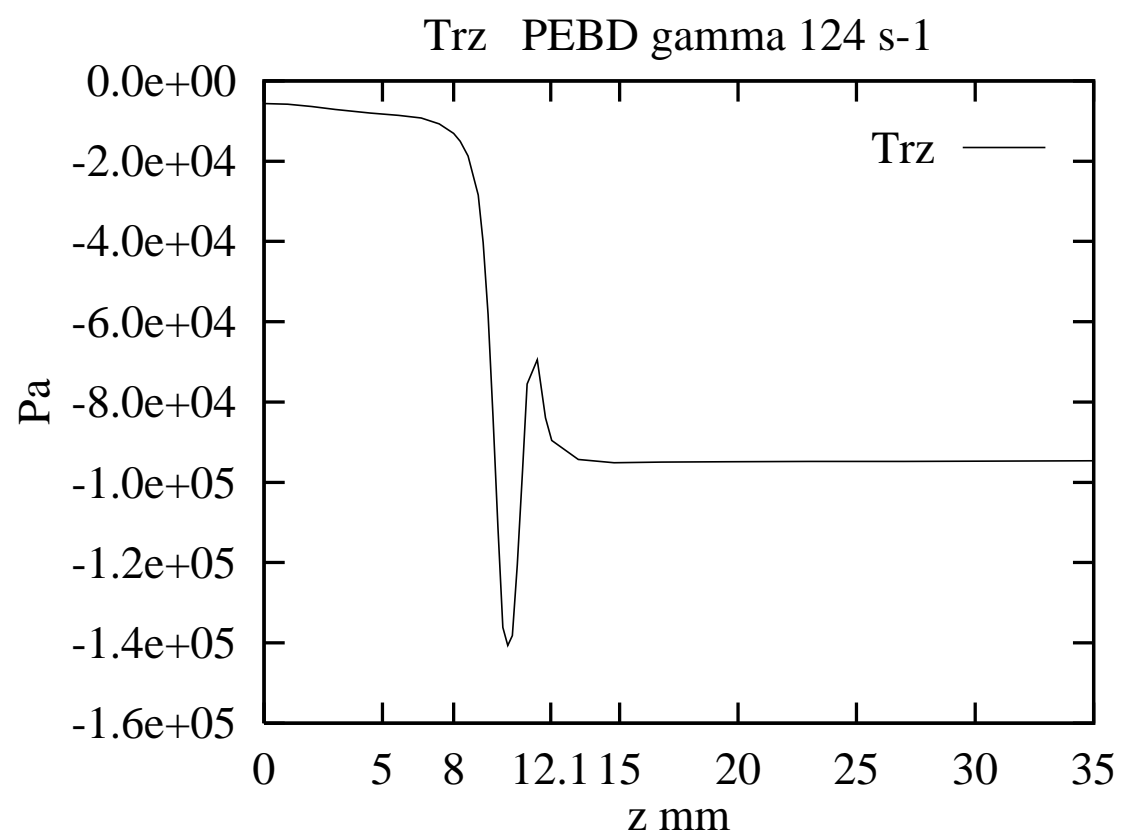

Figure 4. Profil de $T^{r z}$ le long d'une ligne de courant. Cas du PEBD. $\Gamma=124 \mathrm{~s}^{-1}$

\begin{tabular}{|c|c|c|c|c|c|c|c|c|}
\hline \multirow{3}{*}{$\Gamma \mathrm{s}^{-1}$} & \multicolumn{4}{|c|}{ PEBD } & \multicolumn{4}{|c|}{ PEBDL } \\
\hline & \multicolumn{2}{|c|}{$\Delta P_{e} \mathrm{MPa}$} & \multicolumn{2}{|c|}{$\Delta P_{t} \mathrm{MPa}$} & \multicolumn{2}{|c|}{$\Delta P_{e} \mathrm{MPa}$} & \multicolumn{2}{|c|}{$\Delta P_{t} \mathrm{MPa}$} \\
\hline & Num. & Exp. & Num. & Exp. & Num. & Exp. & Num. & Exp. \\
\hline 3.72 & 0.16 & 0.36 & 2.43 & 2.25 & $\overline{0.10}$ & 0.12 & 2.97 & 2.44 \\
\hline 12.38 & 0.31 & 0.77 & 4.16 & 3.82 & 0.37 & 0.32 & 6.94 & 6.01 \\
\hline 37.16 & 0.56 & 1.45 & 6.28 & 6.17 & 0.57 & 0.72 & 13.6 & 11.8 \\
\hline 123.9 & 1.08 & 2.20 & 9.81 & 10.0 & 1.44 & 1.54 & 23.2 & 20.8 \\
\hline
\end{tabular}

TABle 5. Pertes de charge totale et d'entrées. Mesures expérimentales et calculs numériques

Expérimentalement, ces pertes de charge totales sont calculées par la mesure de :

$$
\Delta P_{t}=\sigma_{z_{1}}^{r r}-\sigma_{z_{0}}^{r r}
$$

où $z_{0}$ et $z_{1}$ désignent les sections amont et aval de la filière, $\sigma^{r r}$ la composante normale du tenseur total des contraintes. Les pertes de charge d'entrée sont obtenues par des diagrammes de Bagley [8]. La confrontation entre les valeurs numériques et expérimentales des pertes de charge a été faite pour des filières de rapport de longueur sur diamètre égal à 20, aux quatre taux de cisaillement apparent définis dans le tableau Tab. 4.

Pour les pertes de charge totales, l'accord entre les valeurs expérimentales et numériques est bon, que ce soit pour le PEBD ou le PEBDL (cf. Tab. 5). A l'inverse, pour les pertes de charges d'entrée, cet accord n'est satisfaisant que dans le cas du PEBDL.

$\mathrm{Si}$ les résultats expérimentaux sur les valeurs de ces pertes de charge sont obtenues avec une bonne précision, cette notion de perte de charge à l'entrée constitue sur le plan de la simulation numérique une grandeur globale particulièrement sensible : Elle est associée à un calcul d'écoulement pour une conduite comportant des singularités géométriques. Au voisinage 

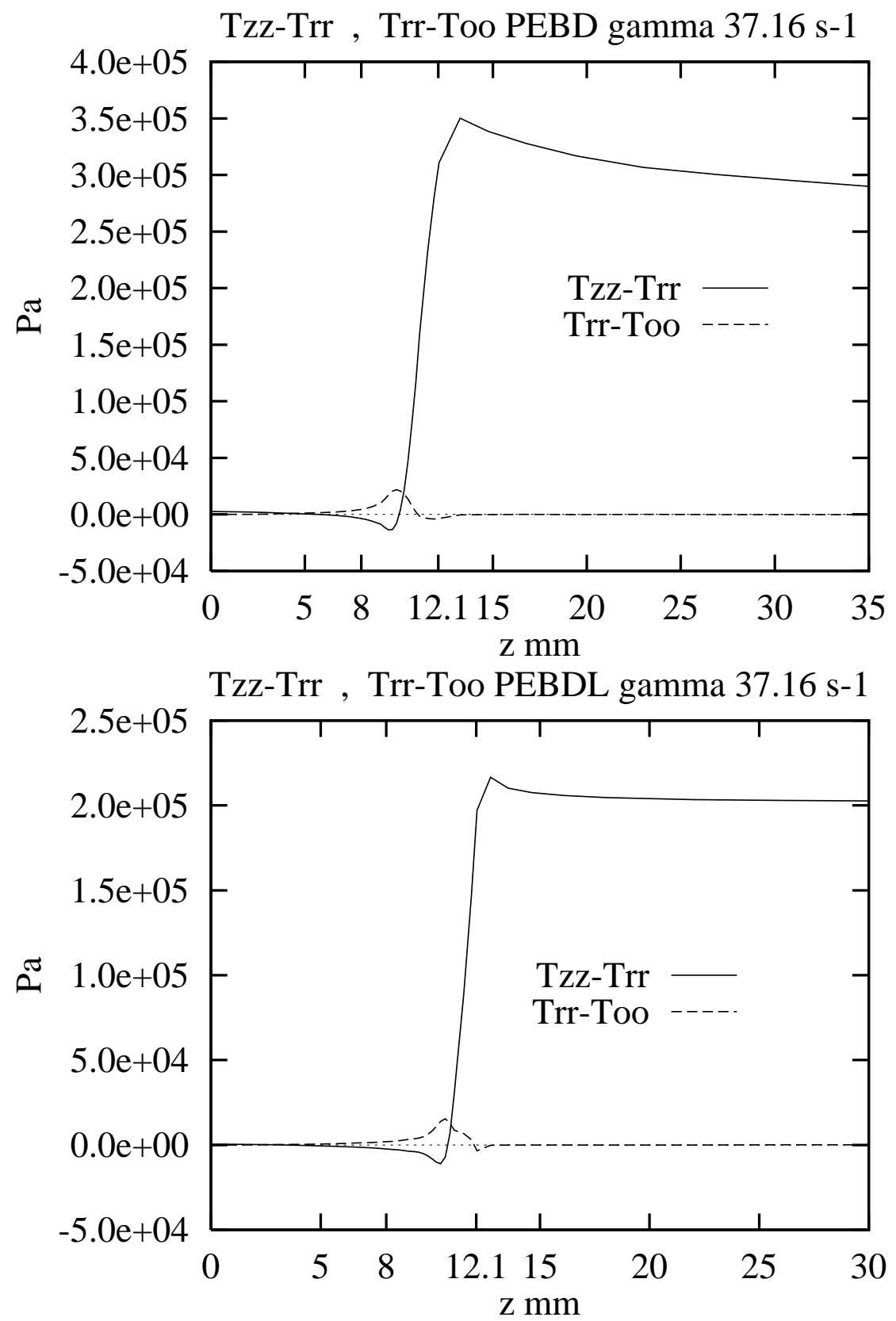

Figure 5. Profil de $T^{z z}-T^{r r}$ et de $T^{r r}-T^{\theta \theta}$ le long d'une ligne de courant. Cas du PEBD et du PEBDL. $\Gamma=37.2 \mathrm{~s}^{-1}$

des sections de singularité, les grandeurs cinématiques sont très importantes et l'écoulement combine des déformations de cisaillement et d'élongation (écoulement mixte). Il peut se poser alors la question de la représentativité du modèle rhéologique dont les paramètres ont été déterminés pour des écoulements simples de cisaillement et d'élongation, dans une certaine gamme de taux de déformation.

En ce qui concerne nos résultats, il est possible de noter que la définition des contraintes directement en fonction des inconnues de la formulation $f$, $f_{R}^{\prime}, f_{Z}^{\prime}$, qui sont elles mêmes relatives à la forme du réseau de lignes de courant, entraînent que toute les variations de ces inconnues au voisinage de la section de contraction se répercutent dans les valeurs des contraintes. 
On peut alors noter que le désaccord entre les valeurs numériques et expérimentales des pertes de charge d'entrée ne s'est produit que pour le PEBD dont les valeurs des différences de contraintes normales sont plus élevées que pour celle du PEBDL, mais celle des contraintes de cisaillement $\mathrm{T}^{r z}$ beaucoup plus faible. De plus, à vitesse d'écoulement égale, le PEBD présente des nombres $S_{R}$ nettement plus élevés que le PEBDL (cf. Tab. 4). Cette répartition différente des niveaux de contraintes selon le type des polymères suggère que le désaccord entre les valeurs expérimentales et numériques des pertes de charge d'entrée est peut être du a une approximation des variables dans la région du convergent insuffisante pour le PEBD mais tout à fait valable pour le PEBDL, le moins "viscoélastique" des deux polymères fondus.

Dans ce contexte, on peut souligner la procédure définie par la Méthode des Tubes de Courant. Comme précisé précédemment, cette méthode permet de considérer un sous-domaine du domaine total, ce qui limite évidemment les différences d'ordre de grandeur des équations et des inconnues, par rapport à des méthodes qui nécessitent une résolution globale dans tout le champ de l'écoulement. Les résultats présentés par d'autres auteurs [6], qui ont développé des modélisations dans le domaine global de l'écoulement, indiquent un désaccord plus important que celui que nous avons obtenu par notre procédure de simulation numérique.

\section{Conclusion}

La Méthode des Tubes de Courant nous a permis de développer une formulation des modèles intégraux codéformationnels. Elle a été ici appliquée dans le cas spécifique des modèles de Wagner et des écoulements en filière convergente axisymétrique. Les solutions numériques ont été obtenues à haut régimes d'écoulement sans difficultés majeures de convergence. La seule considération du tube de courant périphérique a montré des effets viscoélastiques sur les profils de contraintes et sur le réseau des lignes de courant $\mathrm{du}$ domaine d'écoulement principal. L'étude sur les écoulements en convergents axisymétriques était particulièrement pertinente à mener car elle fournissait l'occasion de confronter les résultats de la formulation avec les mesures des pertes de charge d'entrée et totales, et cela pour deux polyéthylènes basse densité PEBD et PEBDL. Dans la gamme des taux de cisaillement apparents que nous avons étudiés, pour ces deux polymères à l'état fondu, nous avons retrouvé un bon accord entre les mesures expérimentales des pertes de charge totales et de leur valeurs calculées numériquement. Pour les pertes de charges d'entrée nous avons également trouvé un bon accord pour le PEBDL, ce qui valide dans ce cas à la fois le modèle et la formulation par la Méthode des Tubes de Courant. Toutefois, un désaccord subsiste entre les valeurs numériques et les mesures expérimentales pour le PEBD. Cette discordance peut être imputée à la complexité des écoulements au voisinage de la singularité et au caractère viscoélastique plus marqué du PEBD.

\section{REFERENCES}

[1] Y. Béreaux, J. R. Clermont. Numerical Simulation of Non-Newtonian Complex Flows Using the Stream-Tube Method and Memory Integral Constitutive Equations. International Journal for Numerical Method in Fluids. 21, pp. 371-389, (1995)

[2] Y. Bereaux. Simulation Numérique d'écoulements de fluides Viscoélastique à formulation intégrale, par la Méthode des Tubes de Courant. Thèse de doctorat INPG (1996). 
[3] J. R.ClERMONT Calculation of kinematic histories in two- and three-dimensional flows using streamline coordinate functions Rheol. Acta. (32) pp. 82-93, 1993

[4] J. R. CleRMONT Sur la modélisation numérique des écoulements plans et méridiens de fluides non-newtoniens incompressibles C. R. Acad. Sc. Paris. t 297 série 2.1, 1983

[5] J. R. Clermont, M. E. De La Lande. Calculation of Main Flows of a Memory Integral Fluid in an Axisymmetric Contraction at High Weissenberg Numbers. J. of Non-Newtonian Fluid Mech. (46), pp. 89-110. (1993)

[6] K. Feigl, H. C. ÖtTingeR. The flow of a LDPE melt through an axisymmetric contraction: A numerical study and comparison to experimental results. J. Rheol. (38-4), p. 847. (1994).

[7] J. Guillet C. Carrot, A. Arsac, P. Revenu Comportement rhéologique en cisaillement et en élongation de deux polyéthylènes. Test de quelques lois constitutives. Cahiers de Rhéologie. (9), nº3-4, Octobre (1993)

[8] J. Guillet, P. Revenu, Y. Bereaux,J.R. Clermont . Experimental and Numerical Study of Entry Flow of Low-Density Polyethylene Melts. Rheol. Acta 35. (1996).

[9] M. H. Wagner. A constitutive analysis of uniaxial elongational flow data of a lowdensity polyethylene melt. J. of Non-Newtonian Fluid Mech. (4), pp. 39-55. (1978)

Laboratoire de RhÉologie (UMR 5520) Domaine Universitaire BP 5338041 GRENOBLE CEDEX 9 FRANCE 